\title{
ARTICLE
}

\section{Recent Developments in Pre-Equilibrium and De-Excitation Models in Geant4}

\author{
José Manuel QUESADA ${ }^{1, *}$, Vladimir IVANCHENKO ${ }^{2,3,4}$, Anton IVANCHENKO $^{2,5}$, \\ Miguel Antonio CORTÉS-GIRALDO ${ }^{1}$, Gunter FOLGER ${ }^{2}$, Alex HOWARD ${ }^{6}$ and Dennis WRIGHT ${ }^{7}$ \\ ${ }^{1}$ Departmento de Física Atómica, Molecular y Nuclear, University of Sevilla , 41080-Sevilla, Spain \\ ${ }^{2}$ CERN, CH-1211 Geneve 23, Switzerland \\ ${ }^{3}$ Ecoanalytica, 119899 Moscow, Russia \\ ${ }^{4}$ Université de Metz, LPMC, 57078 Metz, France \\ ${ }^{5}$ Université de Bordeaux 1, CNRS/IN2P3, CENBG, 33175 Gradignan, France \\ ${ }^{6}$ ETH, Zurich, Switzerland \\ ${ }^{7}$ Stanford Linear Accelerator Center, Menlo Park, California, USA
}

\begin{abstract}
Geant4 native pre-equilibrium and de-excitation models are used for sampling of residual nucleus fragmentation in a number of generators of inelastic hadron/ion interaction with nuclei including QGS, FTF, Binary Cascade, QMD and some others. The pre-compound model is responsible for pre-equlibrium emission of protons, neutrons and light ions. The de-excitation model provides sampling of evaporation of neutrons, protons and light fragments up to magnesium, Fermi break-up, statistical multifragmentation, fission and photon emission. Recently a review of the pre-equilibrium and de-excitation models of Geant 4 has been performed and we report a summary of modifications introduced. The results of the validation versus various published data are presented.
\end{abstract}

KEYWORDS: Geant4, Monte Carlo, hadronic processes, pre-equilibrium, de-excitation, spallation

\section{Introduction}

Geant 4 is a toolkit for the simulation of the passage of particles through matter, initially intended for the preparation of experiments for the Large Hadron Collider. ${ }^{1,2)}$ Nevertheless, its areas of applications are growing and now include nuclear and accelerator physics, spallation reactions, studies in hadron therapy, tomography, space dosimetry, and others. Geant 4 physics includes different models for simulation of interactions of hadrons with nuclei. Geant 4 native pre-equilibrium and de-excitation models are used as backend stages of Binary Cascade (BIC). ${ }^{3)}$ and Quantum Molecular Dynamics model (QMD). ${ }^{4)}$ based generators, which makes them specially suitable for the description of nuclear reactions taking place in spallation and hadron therapy simulations.

In nuclear physics studies, the abundant calculations performed in the past used sets of parameters which were adjusted to fit experimental data. However, we found that by using standard global prescriptions for the model parameters is possible to obtain usually excelent agreement with the data. This fact clearly shows the reliability of such models as well as the global prescriptions for the different magnitudes (inverse cross sections, transition probabilities, etc..).

Geant4 has contributed to the IAEA nuclear spallation reactions benchmark. ${ }^{5,6)}$ with simulation results for all mandatory sets of data, including neutron production, light charged

*Corresponding author, E-mail:quesada@us.es

\footnotetext{
(C) 2011 Atomic Energy Society of Japan, All Rights Reserved.
}

particle production, isotope production, excitation functions up to $3 \mathrm{GeV}$, and pion production. As a consequence of this participation, a series of model improvements have been carried out in Geant 4 native pre-equilibrium and de-excitation models in order to improve their performance. We compare results of such models (as backends of Geant4 Binary Cascade) with predictions of Bertini cascade (BERT), ${ }^{7)}$ which embeds its own pre-compound and de-excitation models, and the experimental data. ${ }^{5}$

\section{Model Review}

\section{Pre-Equilibrium}

Geant4 native pre-equilibrium model is based on the semiclassical exciton model. ${ }^{8}$ The precompound stage of nuclear reaction is considered until nuclear system reaches equilibrium. During this stage, transitions to states with different number of excitons compete with particle emissions, including emission of light coumpound fragments (up to alpha). Further emission of nuclear fragments or photons from excited nucleus (as well as fission and nucleus explosive break-up's) is simulated using equilibrium de-excitation models.

The transition to the state of statistical equilibrium is characterized by an equilibrium number of excitons $n_{e q}$, when all types of transitions are equiprobable. Thus $n_{e q}$ is fixed by

$$
\lambda_{+2}\left(n_{e q}, E^{*}\right)=\lambda_{-2}\left(n_{e q}, E^{*}\right),
$$

where $\lambda_{ \pm 2}$ is the transition rate to a state with \pm 2 excitons and 
$E^{*}$ is the excitation energy. The transition probabilities (a key ingredient of the model) have been calculated either in a semimicroscopical way. ${ }^{9)}$ or using a standard parameterization of the matrix elements. ${ }^{10)}$ In the case of double-differential spectra of particle emission, both prescriptions lead to similar results, but when trying to reproduce isotope production in typical spallation reactions, ${ }^{11)}$ the semi-microscopical transition probabilities. ${ }^{9)}$ produce better results. Therefore, the last one has been chosen as default. From Eq. (1) one can get as a very rough estimate

$$
n_{e q}=\sqrt{2 g E^{*}}
$$

where $g$ is the single-particle level density.

In practice, the factor 2 inside the square root of the rhs of Eq. (2) is considered an adjustable parameter in nuclear data evaluations. We have found that one must be very cautious with this estimate since it becomes evident that in certain circumstances (heavy targets at bombarding energies where pre-equilibrium plays a role) condition (1) is reached far before $n=n_{e q}$ and therefore the former must prevail. In our Monte Carlo simulation, this physically-consistent condition has been directly implemented by means of the apropriate algorithm.

The inverse reaction cross section is another key ingredient in the calculation of particle emission probabilities. Initial formulation ${ }^{12)}$ was previous to the wealth of experimental data since the sixties. Therefore, several parameterizations, either of experimental cross sections ${ }^{13)}$ or of calculated cross sections from optical potentials in turn fitted to the available nuclear reaction data sets, ${ }^{14,15)}$ have been included as options.

\section{De-Excitation}

Geant4 native de-excitation model includes several semiclassical alternative/competitor models:

- evaporation of nucleons and light fragments either

- up to $\alpha$ particles, based on Weisskopf-Ewing ${ }^{16)}$ model or

- up to ${ }^{28} \mathrm{Mg}$, based on Generalized Evaporation Model (GEM), ${ }^{17)}$

- evaporation of photons:

- discrete (according to tabulated E1, M1 and E2 transition probabilities) and

- continuous (according to E1 giant dipole resonance strenght distribution),

- fission, based on Bohr-Wheeler semi-classical model, ${ }^{18)}$

- statistical multifragmentation, ${ }^{19)}$

- Fermi break-up. ${ }^{19)}$

Figure 1 shows the main ingredients of the current implementation in the de-excitation handler class.

As previously stated, recent contribution to the IAEA nuclear spallation reactions benchmark has triggered a review of the native low energy nuclear models of Geant 4 , specially at

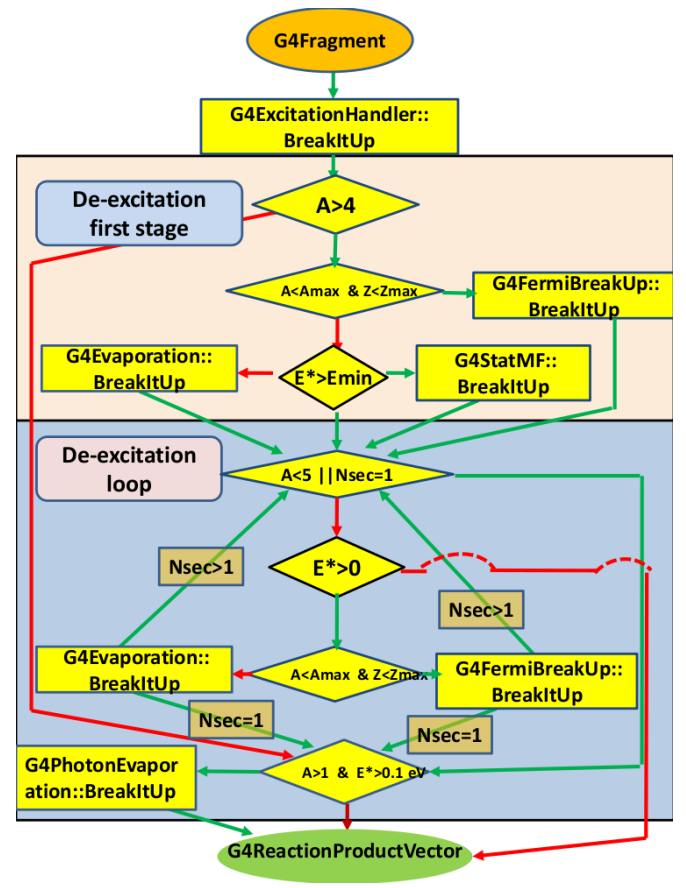

Fig. 1 Basic de-excitation diagram in v9.3. Nsec stands for the number of secondaries.

equilibrium de-excitation stage. Initially it was evident from the analysis of the isotope production experimental data that there were problems in the Geant4 latest release at that time (4.9.2p01), since

- the IMF (Intermetiate Mass Fragment, light nuclei with $2<\mathrm{Z} \leq 12$ and $\mathrm{A} \leq 28$ ) production could not be reproduced,

- the implementation of fission model failed to reproduce the height and shape of the fission bell,

- the slope of the spallation region (shoulder for heavier fragments) was too steep.

Initial excited nuclei are too heavy for Fermi break-up $\left(\mathrm{A}_{\max }^{F B U}=16, \mathrm{Z}_{\max }^{F B U}=8\right)$ and too cold to undergo statistical multi-fragmentation $\left(\mathrm{Ex}_{\text {min }}^{* S M F}=3 \mathrm{MeV}\right)$; therefore the generalized evaporation model ${ }^{17)}$ is the only candidate to accomplish this task. Nevertheless, an intensive development work was required in order to make it operational. After it, surprisingly good agreement with experimental data has been achieved in the IMF region (Figs. 2-5).

With regard to the second point, one should consider an important aspect pointed by the Los Alamos Group working in MCNPX code: ${ }^{20)}$ many models, each if them containing phenomenological parameters, are currently being used in environments which are different to their original ones, i.e. in competition with different de-excitation mechanisms. Moreover, the energy distribution in the initial population of excited nuclei, which changes greatly from one model to another (QMD and BIC, for instance) is of critical importance for the de-excitation process. Therefore there is no reason to expect that these effective values of the parameters would produce 
good results when used in different scenarios. According to these ideas we have performed a small parameter tuning of fission parameters:

- level density parameter ratio $a_{f i s} / a_{\text {evap }}$,

- width of symmetric component of the fission,

which turned out to produce reasonably good results (Figs. 35).

As to the third aspect, a possible cure for it will be discussed in the next Results subsection, as an ongoing development, not yet included in the official release.

The new inverse reaction cross sections ( fitted to experimental data either directly or indirectly through optical model global fittings) improve the description of the evaporation of neutrons and light charged ions with $\mathrm{Z} \leq 2$, when described in the framework of Weisskopf-Ewing standard evaporation model. This is an advantage with respect to the use of the much older Dostrovsky's parameterization, which, on the other hand, allows analytic integration . Nevertheless, in GEM the later is used and the inclusion of the former ones would ask for numerical integration which in turn would have a big impact in CPU time consumption (since many fragments are considered, including excited ones). In order to preserve the improved description of neutron and light charged particle emission with standard evaporation model, while using GEM for the heavier ones, a new hybrid model has been implemented, which uses :

- Weisskopf-Ewing standard evaporation model for the emission neutrons and light charged ions with $\mathrm{Z} \leq 2$, with improved inverse reaction cross sections,

GEM for heavier fragments $(\mathrm{Z} \leq 12$ and $\mathrm{A} \leq 28)$.

\section{Results}

A selection of the results obtained in the IAEA nuclear spallation reactions benchmark are presented in Figs. 2-5 and Figs. 6-11. They have been calculated with the official release of Geant4, version 9.3p01.

As a typical limitation of cascade and pre-equilibrium models (which has been put in evidence in the IAEA benchmark. ${ }^{5)}$ ), coalescence mechanism is clearly missing in our simulations (see Figs. 9 and 10). Development effort is foreseen in this aspect.

As already mentioned in previous De-excitation subsection, there is still room for additional refinements, specially at the deep spallation region ( shoulder at the right side of the residue production plots, see Figs 3-5). A smooth transition around $n_{e q}$ from preequilibrium to equilibrium was initially set into the model ${ }^{21)}$ (soft cutoff criterium) in order to enhance evaporation at the expense of preequilibrium. In our previous analyses of neutron emission spectra from proton induced reactions, ${ }^{22)}$ this mechanism had proven to worsen the results. Nevertheless, our recent studies in the IAEA nuclear spallation reactions benchmark (in particular, residue production) showed that the soft cutoff mechanism is necessary to some extent. A good compromise turned out to be a more consistent version of the soft cutoff algorithm ( $n_{e q}$ being strictly calculated from condition (1)), which allows for a much smaller diffusivity of the soft cutoff region around it. The price to pay is a penalty in CPU time, since for each initial excited nucleus, before the full chain of pre-equilibrium transitionversus-emission steps is made, a dummy transition loop (without particle emissions) must be performed until equilibrium condition (1) is accomplished. This improves the description of residue production experimental data of spallation reactions (Figures 12 and 13) without sizeable deterioration of neutron spectra. This new prescription is still under testing and has not yet been included in the official release of Geant 4 .

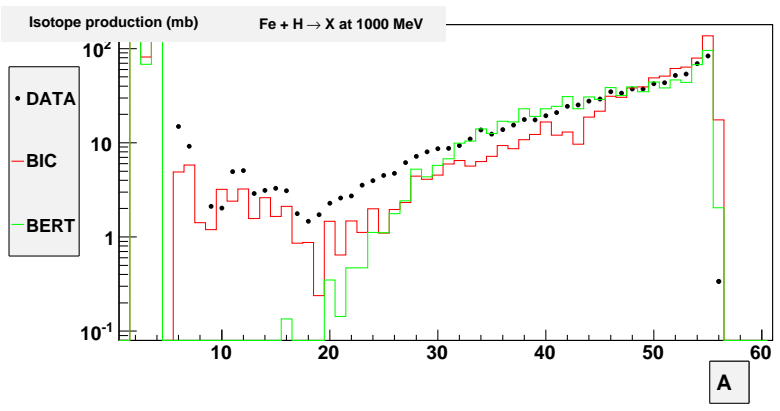

Fig. $2 \mathrm{p}+\mathrm{Fe}$ at $1 \mathrm{GeV}$, residue production

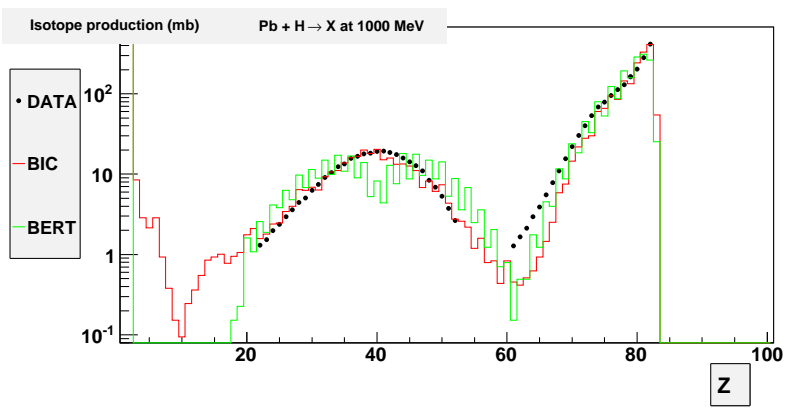

Fig. $3 \mathrm{p}+\mathrm{Pb}$ at $1 \mathrm{GeV} / \mathrm{A}$ (inverse kinematics) residue production

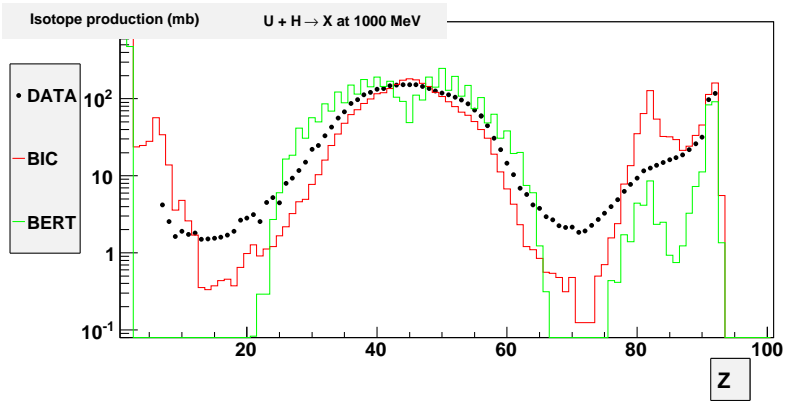

Fig. $4 \mathrm{p}+\mathrm{U}$ at $1 \mathrm{GeV} / \mathrm{A}$ (inverse kinematics) residue production 


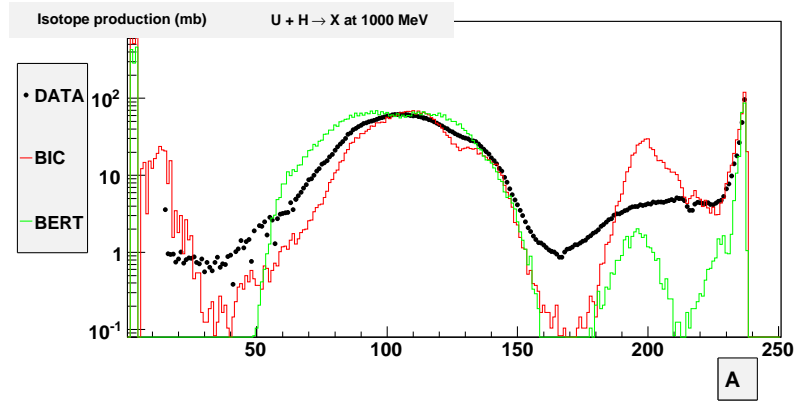

Fig. $5 \mathrm{p}+\mathrm{U}$ at $1 \mathrm{GeV} / \mathrm{A}$ (inverse kinematics) residue production

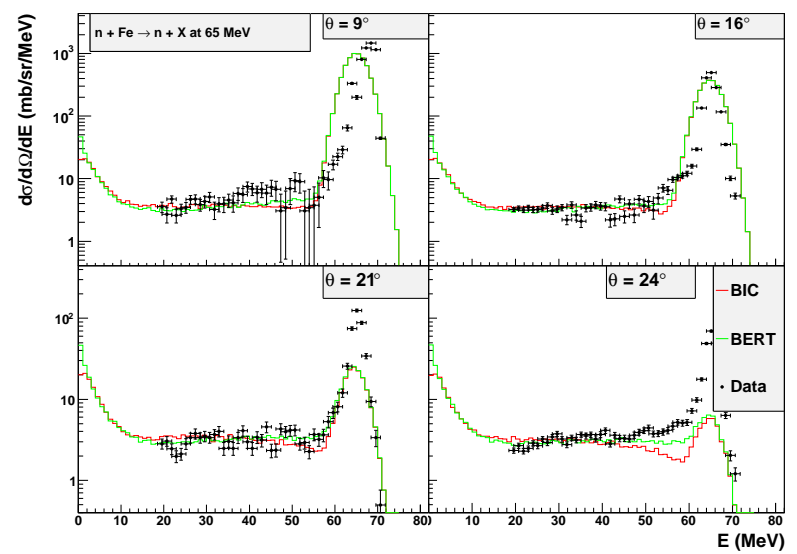

Fig. $6 \mathrm{n}+\mathrm{Fe}$ at $65 \mathrm{MeV}$, neutron production

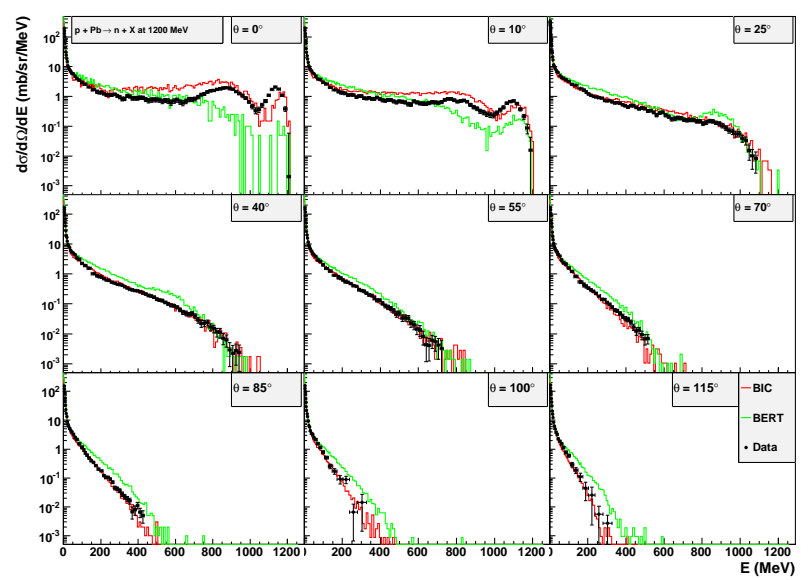

Fig. $7 \mathrm{p}+\mathrm{Pb}$ at $1,200 \mathrm{MeV}$, neutron production

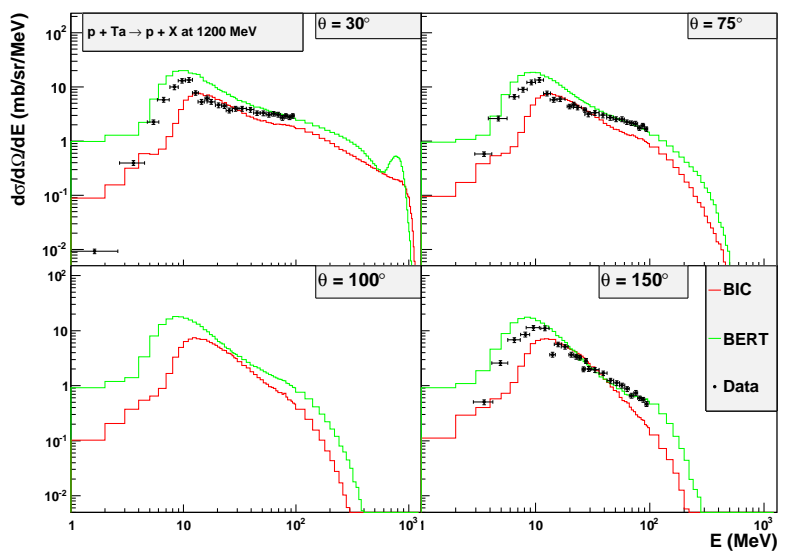

Fig. 8 p+Ta at $1.2 \mathrm{GeV}$, proton production

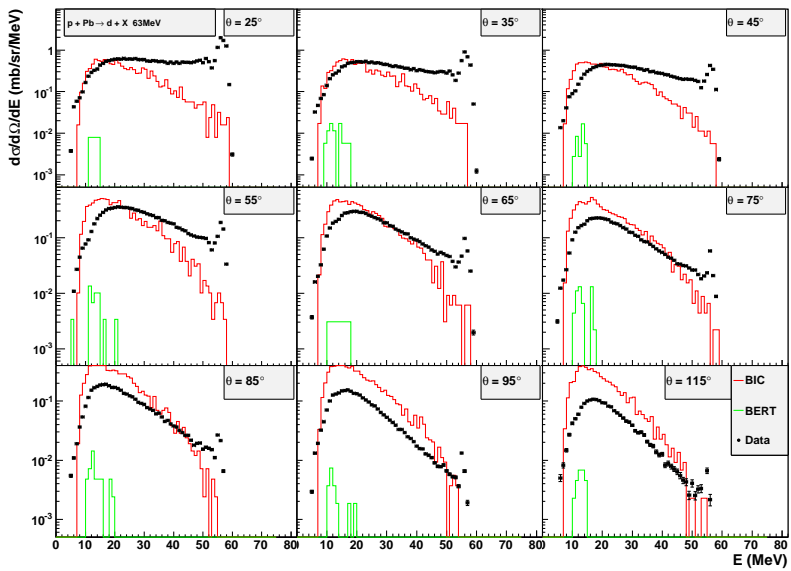

Fig. $9 \mathrm{p}+\mathrm{Pb}$ at $63 \mathrm{MeV}$, deuteron production

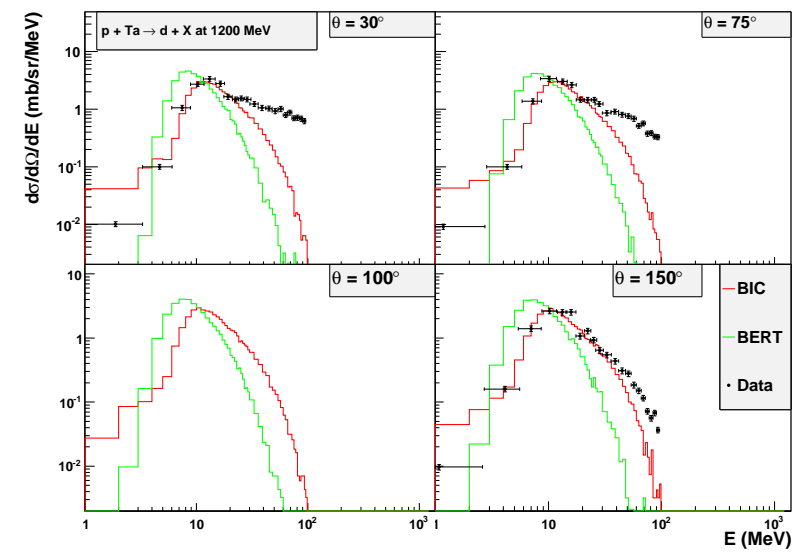

Fig. $10 \mathrm{p}+\mathrm{Ta}$ at $1.2 \mathrm{GeV}$, deuteron production 


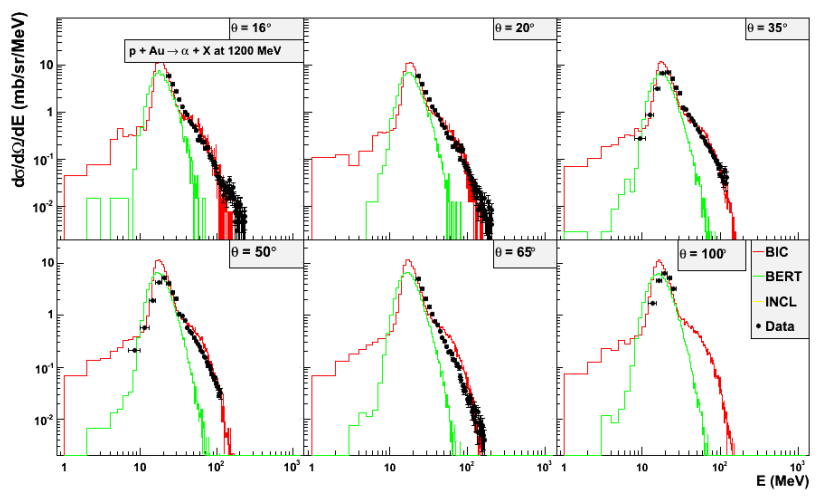

Fig. $11 \mathrm{p}+\mathrm{Au}$ at $1.2 \mathrm{GeV},{ }^{4} \mathrm{He}$ production

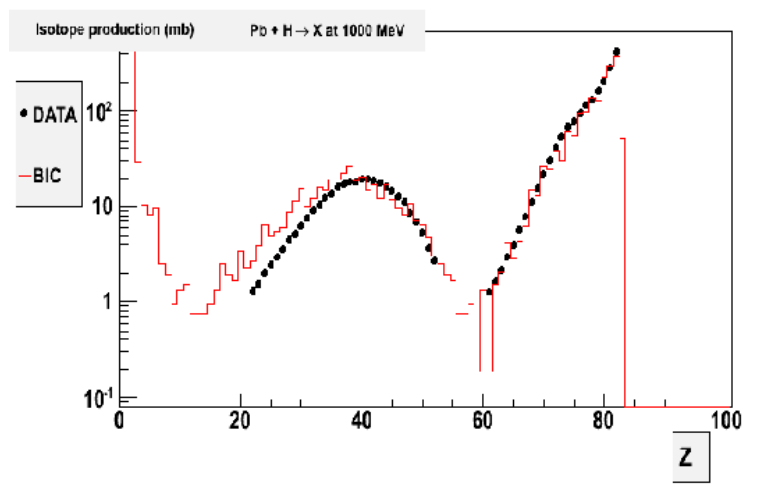

Fig. 12 Improved: $\mathrm{p}+\mathrm{Pb}$ at $1 \mathrm{GeV} / \mathrm{A}$ (inverse kinematics) residue production. Deep spallation region (shoulder at the right side of the plot) can be better reproduced after ad hoc parameter tuning

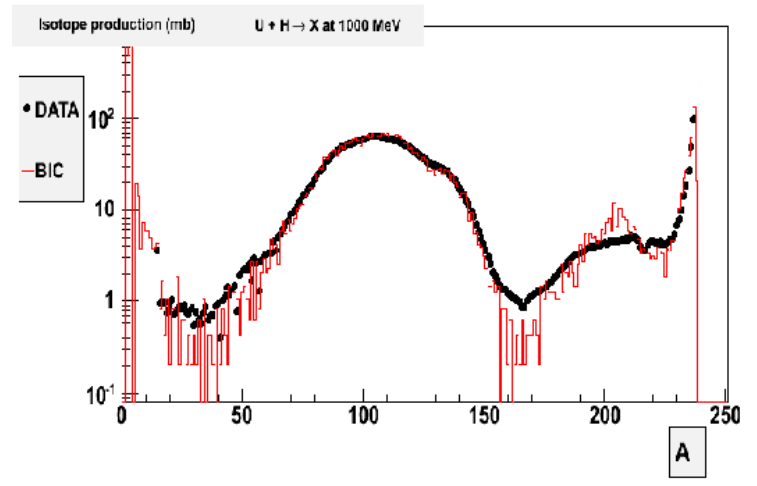

Fig. 13 Improved: $\mathrm{p}+\mathrm{U}$ at $1 \mathrm{GeV} / \mathrm{A}$ (inverse kinematics) residue production. Same comments as in previous figure apply here

\section{Conclusion}

The review of the native pre-equilibrium and de-excitation models of Geant 4 recently performed has led to an overall satisfactory reproduction of experimental data set of IAEA nuclear spallation reactions benchmark. Additional development work is in progress.

\section{Acknowledgment}

The authors would like to thank their co-workers in the Geant4 Hadronic Working Group.

This work was partially supported by the spanish Ministerio de Educación y Ciencia (under contracts FPA2008-04972C03-02 and FIS2008-04189) and European Space Agency (under ESA TRP contract 22712/09/NL/AT).

\section{References}

1) S. Agostinelli et al., "Geant4 - a simulation toolkit",Nucl. Instr. Meth. Phys. Res., A506, 250-303 (2003).

2) J. Allison et al., "Geant4 developments and applications," Nucl. Sci. IEEE Trans., 53[1], 270-278 (2006).

3) G. Folger, V. N. Ivanchenko, H. P. Wellisch, "The Binary Cascade - nucleon-nuclear reactions," Eur. Phys. J., A21, 407-417 (2004).

4) T. Koi, "New Native QMD Code in Geant4," Proc. IEEE 2008 Nuclear Science Simposium, Dresden, Germany, Oct. 19-25, 2008 (2008), [CD-ROM].

5) IAEA Benchmark of Spallation Models, http://nds121.iaea.org/alberto/mediawiki-1.6.10/index.php

6) J. Apostalakis, A. Ivantchenko, V. N. Ivanchenko, M. Kossov, J. M. Quesada, D. H. Wright, "Geant4 simulation of nuclear spallation reactions," Proc. International Topical Meeting on Nuclear Research Applications and Utilization of Accelerators. Satellite Meeting on Spallation Reactions, IAEA, Vienna, Austria, May 4-8 (2009), [CD-ROM].

7) A. Heikkinen, N. Stepanov, H. P. Wellisch, "Bertini intranuclear cascade implementation in Geant4," Proc. CHEP'03, La Jolla, California, USA, March 24-28 (2003).

8) J. J. Griffin, "Statistical Model of Intermediate Structure," Phys. Rev. Lett., 17, 478-481 (1966).

9) K. Gudima, S. G. Mashnik, V. D. Toneev, "Cascade-exciton Model of Nuclear Reactions," Nucl. Phys., A401, 329-361 (1983).

10) S. Gupta, "Two-component equilibration in the exciton model of nuclear reactions," Z. Phys., A303[4], 329-333 (1981).

11) J. M. Quesada, on behalf of the Geant 4 Hadronic Working Gropup, "Results obtained with nuclear models of Geant4 in IAEA Benchmark of Spallation," Second Advanced Workshop on Model Codes for Spallation Reactions

http://nds121.iaea.org/alberto/mediawiki-1.6.10/index.php/ Benchmark:2ndWorkProg, CEA-Saclay (France), 8-11 February (2010).

12) I. Dostrovsky, Z. Fraenkel, G. Friedlander, "Monte Carlo Calculations of Nuclear Evaporation Processes. III. APllications to Low-Energy Reactions," Phys. Rev., 116[3], 683-702 (1959).

13) H. P. Wellisch, D. Axen, "Total reaction cross section calculations in proton-nucleus scattering," Phys. Rev., C54[3], 13291332 (1996).

14) A. Chaterjee, K. H. N. Murthy, S. K. Gupta, Pramana, 16[5], 391-402 (1981). 
15) C. Kalbach, PRECO-2000 Exciton Model Preequilibrium Code with Direct Reactions, NEA Data Bank.

16) V. E. Weisskopf, D. H. Ewing, "On the Yield of Nuclear Reactions with Heavy Elements," Phys. Rev., 57, 472-485 (1940).

17) S. Furihata, K. Niita, S. Meigo, Y. Ikeda, F. Maekawa, The GEM Code - A Simulation Program for the Evaporation and Fission Process of an Excited Nucleus -, JAERI-Data/Code 2001-015, Japan Atomic Energy Research Institute (JAERI) (2001).

18) N. Bohr, J. A. Wheeler, "The Mechanism of Nuclear Fission," Phys. Rev., 56, 426-450 (1939).

19) J. P. Bondorf, A. S. Botvina, A. S. Iljinov, I. N. Mishustin, K. Sneppen, "Statistical multifragmentation of nuclei," Phys. Rep., 257, 133-221 (1995).

20) S. G. Mashnik, K. K. Gudima, R. E. Prael1, A. J. Sierk, M. I. Baznat, N. V. Mokhov, CEM03.03 and LAQGSM03.03 Event
Generators for the MCNP6, MCNPX, and MARS15 Transport Codes, LA-UR-08-2931, Los Alamos National Laboratory (LANL) (2008)

21) S. G. Mashnik, A. J. Sierk, K. K. Gudima, M. I. Baznat, "CEM03 and LAQGSM03 - new modeling tools for nuclear applications", Proc. EPS Euroconference XIX Nuclear Physics Divisional Conference: New Trends in Nuclear Physics Applications and Technology, Pavia, Italy, 5-9 September 2005, J. Phys. Conf. Ser., 41, 340-351 (2006).

22) J. M. Quesada Molina, M. A. Cortés Giraldo, A. Howard, G. Folger, V. N. Ivanchenko, "Improvements of Preequilibrium and Evaporation Models in Geant4," Proc. IEEE Nuclear Science Symposium, Dresden, Germany, Oct. 19-25, 2008, [CD-ROM]. 